The first part of this statement follows immediately from Lagrange's theorem, if it is applied to any closed circuit of electrons starting simultaneously from the cathode, as at the cathode surface the tangential velocity is zero. In order to prove the second part, assume that the normal $n$ to the cathode surface coincides with the $x$ direction, and the tangential component of $H$ with the $z$ direction. The $y$ component of Lorentz's (1) is

$$
\begin{aligned}
m \frac{d}{d t} v_{y} & =m\left(\frac{\partial v_{y}}{\partial t}+v_{x} \frac{\partial v_{y}}{\partial x}+v_{y} \frac{\partial v_{y}}{\partial y}+v_{z} \frac{\partial v_{y}}{\partial z}\right) \\
& =-e E_{y}+\frac{e}{c} v_{x} H_{z} .
\end{aligned}
$$

At the cathode surface

$$
v_{y}=v_{z}=\frac{\partial v_{y}}{\partial t}=E_{y}=0
$$

These terms go to zero at the cathode surface at least like $x$, whereas the remaining terms which have $v_{x}$ as a factor go to zero like $x^{1 / 2}$ at a saturated cathode and like $x^{2 / 3}$ if the discharge is space-charge-limited. Thus the remaining terms are dominant near the cathode surface. Dividing these by $v_{x}$ we obtain

$$
m \frac{\partial v_{y}}{\partial x}=\frac{e}{c} H_{z} \text {. }
$$

On the other hand at the cathode surface, as $v_{x}=0$

Thus

$$
\operatorname{curl}_{z} \mathbf{v}=\frac{\partial v_{y}}{\partial x}-\frac{\partial v_{x}}{\partial y}=\frac{\partial v_{y}}{\partial x}
$$

$$
\begin{aligned}
\operatorname{curl}_{z} \mathbf{p} & =\operatorname{curl}_{z}\left(m \mathrm{v}-\frac{e}{c} \mathbf{A}\right) \\
& =\operatorname{curl}_{z} m \mathrm{v}-\frac{e}{c} H_{z}=0
\end{aligned}
$$

that is to say, the tangential component of the vorticity of the total momentum at the surface of a cathode is zero in all circumstances.

\title{
The Plane-Wave Resolution of Guided Waves*
}

\author{
S. S. MACKEOWN $\dagger$, FELLOW, I.R.E., AND JOHN W. MILES $\ddagger$
}

Summary-Wave propagation in cylindrical guides of both rectangular and circular cross section is treated by representing the proper solutions to Maxwell's equation through a plane-wave expansion of the Hertzian vector. In the case of a rectangular wave guide only a finite number of plane waves (two or four) is required to represent a given mode, while for the circular guide an infinite manifold is required. The plane waves are uniform, traveling with the velocity of light in the medium at an angle to the cylindrical axis which is determined by the frequency and the eigenvalue of the mode under consideration.

\section{INTRODUCTION}

$\mathrm{T}$ $\checkmark H E$ PROPOSITION that any solution to the wave equation may be resolved into an expansion of plane waves has been enunciated by several writers, including Stratton ${ }^{1}$ and Ramo and Whinnery. ${ }^{2}$ For academic purposes it nevertheless seems worth while to produce the expansions explicitly for the important cases of propagation in circular and rectangular wave guides. These expansions and concepts have proved helpful in classroom presentation of the subject, particularly in clarifying the concepts of phase and group velocities.

\section{The Field Equations}

In the interests of simplicity we shall derive all field vectors from the Hertzian vector, following Stratton. ${ }^{1}$

* Decimal classification: R116. Original manuscript received by the Institute, February 6, 1945; revised manuscript received, April 16, 1945.

$\dagger$ California Institute of Technology, Pasadena, Calif.

$\ddagger$ Lockheed Aircraft Corporation, Burbank, California.

I J. A. Stratton, "Electromagnetic Theory," McGraw-Hill Book Co., New York, N. Y., 1941.

2 Simon Ramo and John Whinnery, "Fields and Waves in Modern Radio," John Wiley and Sons, New York, N. Y., 1944.
In cylindrical co-ordinates $(u, v, z)$, the fields are completely specified by the $z$ component of the Hertzian vector which is herein represented by the scalar function $\psi(u, v, z)$. ( $z$ is the co-ordinate measured along the axis of the cylinder, and $u$ and $v$ are orthogonal co-ordinates in a plane of constant z.) Inasmuch as the Hertzian vector satisfies the vector wave equation and the $z$ co-ordinate is measured in a fixed direction, its $z$ component must satisfy the scalar wave equation; viz.,

$$
\nabla^{2} \psi+k^{2} \psi=0, \quad k=(\mu \epsilon)^{1 / 2} \omega=2 \pi / \lambda
$$

where $\mu$ and $\epsilon$ are the permeability and the dielectric constant, respectively, $\omega$ is the angular frequency, and $\lambda$ is the wave length in the medium (not, however, the guide wave length). The operator $\partial / \partial t$ has been replaced by $j \omega$ in accordance with the assumption of harmonic time variation. ${ }^{3}$

The scalar and vector potentials $\phi$ and $\bar{A}$ are then given by ${ }^{4}$

$$
\begin{aligned}
\phi & =-\partial \psi / \partial z \\
\bar{A} & =j \omega \mu \epsilon \bar{k} \psi .
\end{aligned}
$$

In the case of $T M$ or $E$ waves; i.e., $H_{z}=0$, we have

$$
\begin{aligned}
& \bar{E}_{E}=-\nabla \phi_{E}-j \omega \bar{A}_{E} \\
& \bar{B}_{E}=\nabla \times \bar{A}_{E}
\end{aligned}
$$

while for $T E$ or $H$ waves $\left(E_{z}=0\right)$ we have

$$
\begin{aligned}
& \bar{D}_{H}=-\nabla \times \bar{A}_{H} \\
& \bar{H}_{H}=-\nabla \phi_{H}-j \omega \bar{A}_{H} .
\end{aligned}
$$

The boundary conditions require the vanishing of the

${ }^{3}$ Note that Stratton assumes a time variation $e^{-i \omega t}$ where we have assumed $e^{j \omega t}$. Simply substituting $-j$ for $i$ reconciles the two assumptions, $e^{j \omega t}$ being more general engineering usage.

${ }^{4}$ See Appendix A for a resume of the vector notation used herein. 
tangential electric field or, equivalently, of the normal magnetic field at the metal surface. For the $E$ modes we may require $\left(\nabla \times \bar{A}_{E}\right) \cdot \bar{n}$ to vanish, ${ }^{5}$ which, from (3) is effected by the vanishing of the tangential derivative of $\psi_{E}$; viz.,

$$
\partial \psi_{E} / \partial s=0
$$

while for the $H$ modes we require $\left(\nabla \times \bar{A}_{H}\right) \cdot \bar{s}$ to vanish, which from (3) is effected by the vanishing of the normal derivative of $\psi_{H}$; viz.,

$$
\partial \psi_{H} / \partial n=0
$$

In treating (1) it is customary to assume propagation along the $z$ axis with a propagation constant $j h$ so that we may write

$$
\psi(u, v, z)=f(u, v) e^{\mp j h z}
$$

where the positive or negative sign is associated with propagation in the positive or negative $z$ direction, respectively. We remark that the mathematical motivation of this choice is one of simplicity, and is not necessarily accompanied by any a priori conviction as to the direction of travel of any individual wave front. The wave represented in (10) has a phase velocity $(\omega / h)$. Substituting (10) in (1) we have the two-dimensional wave equation

$$
\nabla^{2} f+\kappa^{2} f=0, \quad \kappa^{2}=k^{2}-h^{2}
$$

There is a doubly infinite set of solutions to (11) corresponding to a doubly infinite set of discrete values for $\kappa$, these eigenvalues being determined by the boundary conditions (8) or (9).

\section{The Rectangular Wave Guide}

The solutions to (1), satisfying the boundary conditions (8) and (9) in a rectangular wave guide bounded by the planes $x=0, x=a, y=0$, and $y=b(u=x, v=y)$, are found to be (see Appendix B)

$$
\begin{aligned}
\psi_{E_{m n}}(x, y, z) & =\sin (m \pi x / a) \sin (n \pi y / b) e^{\mp j h z} \\
\psi_{H_{m n}}(x, y, z) & =\cos (m \pi x / a) \cos (n \pi y / b) e^{\mp j h z} \\
\kappa_{E^{2}} & =\kappa_{H}{ }^{2}=(m \pi / a)^{2}+(n \pi / b)^{2}
\end{aligned}
$$

where $m$ and $n$ are integers. We observe that, by elementary trigonometry, both (12) and (13) may be obtained by superimposing solutions to (1) of the type

$$
\psi_{m n}(x, y, z)=e^{j[ \pm m \pi(x / a) \pm n \pi(y / b) \pm h z]}
$$

where any combination of the signs is permitted. If we define the angles

we may write

$$
\begin{aligned}
& \alpha=\tan ^{-1}(\kappa / h)=\sin ^{-1}(\kappa / k) \\
& \beta= \pm \tan ^{-1}(m b / n a)
\end{aligned}
$$

$\bar{K}=[\bar{\imath} \sin \alpha \cos \beta+\bar{\jmath} \sin \alpha \sin \beta+\bar{k} \cos \alpha] k$

$\bar{R}=\bar{i} x+\bar{j} y+\bar{k} z$

$\bar{K}$ being the "vector-propagation constant" and $\bar{R}$ the radius vector. The function given by (18) represents a uniform plane wave traveling at a polar angle $\alpha$ to the $z$ axis and an azimuthal angle $\beta$ to the $X$ axis with the velocity of light $\left(c=(\mu \epsilon)^{-1 / 2}\right)$ in the medium. The

\footnotetext{
5 $\bar{n}$ is the unit vector normal to the cylindrical surface in question;
} similarly $\bar{s}$ is the unit tangential vector in a plane of constant $z$. properties of a plane wave, listed in any standard test, ${ }^{1,2}$ include the mutual orthogonality of the electric field, the magnetic field, and the axis of propagation and, for a uniform plane wave, the uniformity of the fields in any plane transverse to the axis of propagation. From (16) we observe that the phase velocity $(\omega / h)$ of any mode is greater than the velocity of light by a factor of $\sec \alpha$. Accordingly, the measured guide wavelength is $\sec \alpha$ greater than $\lambda$, inasmuch as it is measured between planes of constant phase; however, since the plane waves which make up the guided wave travel at an angle $\alpha$ to the $z$ axis, the group velocity of the guided wave is less than the velocity of light by a factor of $\cos \alpha$.

Chu and Barrow, ${ }^{6}$ following Brillouin and Page and Adams, have given the plane-wave resolution for the special case of the $T E_{01}$ mode, where $b$ is greater than $a$, and mention the experimentally observed phenomenon that radiation through a slot cut in one face of the guide is at the predicted angle $\alpha=\sin ^{-1}(\lambda / 2 b$ ) (see (14) and (16) for $m=0, n=1$ ) to the $z$ axis. As a more explicit example of a plane-wave resolution in a rectangular guide, we choose the $H_{m n}$ mode traveling along the positive $z$ axis; from (13) we may write

$$
\begin{aligned}
\psi_{H_{m n}}(x, y, z)= & 1 / 4\left[e^{-j(m \pi x / a+n \pi y / b+h z)}\right. \\
& +e^{-j(-m \pi x / a+n \pi y / b+h z)} \\
& +e^{-j(-m \pi x / a-n \pi y / b+h z)} \\
& \left.+e^{-j(m \pi x / a-n \pi y / b+h z)}\right] \\
= & 1 / 4\left[\psi_{1}+\psi_{2}+\psi_{3}+\psi_{4}\right]
\end{aligned}
$$

where $\psi_{1}, \psi_{2}, \psi_{3}$, and $\psi_{4}$ are plane waves traveling at polar and azimthal angles

$$
\begin{gathered}
\alpha=\sin ^{-1}\left\{\left[(m / a)^{2}+(n / b)^{2}\right]^{1 / 2} \lambda / 2\right\} \\
\beta_{4}^{1}= \pm \tan ^{-1}(m b / n a), \quad \beta_{3}^{2}=\pi \mp \beta_{1}
\end{gathered}
$$

respectively. We remark that, for the special case $m=0 \quad \psi_{1}=\psi_{4}\left(\beta_{1}=\beta_{4}=0\right)$ and $\psi_{2}=\psi_{3}\left(\beta_{2}=\beta_{3}=\pi\right)$, while for $n=0 \quad \psi_{1}=\psi_{2}\left(\beta_{1}=\beta_{2}=\pi / 2\right)$ and $\psi_{3}=\psi_{4}\left(\beta_{3}=\beta_{4}=3 \pi / 2\right)$.

\section{Circular Guide}

For the case of the circular guide we use a treatment which is suggested by Stratton's general treatment of cylindrical waves. ${ }^{1}$ The solutions to (1) in the co-ordinates $(u=r, v=\theta, z)$ satisfying the boundary conditions (8) and (9) in a circular guide of radius $a$ are found to be (see Appendix B)

$$
\begin{aligned}
\psi_{m n}(r, \theta, z)=J_{m}\left(\kappa_{n} r\right) e^{j( \pm m \theta+\phi \mp h z)} & =f(r, \theta) e^{\mp j h z} \\
J_{m}\left(\kappa_{n}{ }^{E} a\right) & =0 \\
J_{m}^{\prime}\left(\kappa_{n}{ }^{\boldsymbol{H}} a\right) & =0
\end{aligned}
$$

where $\operatorname{Jm}(x)$ is the Bessel function of the $m^{\prime}$ th order. ${ }^{7}$ In general, several solutions of the type (23) will have to be linearly combined to give the correct polarization in $\theta$. By, expressing the Bessel function as the contour inte$\mathrm{gral}^{8}$ and making the change of variable $\beta=\phi \pm \theta$

- L. J. Chu and W. L. Barrow, "Electromagnetic waves in hollow metal tubes of rectangular cross section," PROC. I.R.E., vol. 26, pp. 1520-1555; December, 1938.

${ }^{7} \phi$ is an arbitrary phase constant specifying the polarization of the potential.

${ }^{8}$ Eugene Jahnke and Fritz Emde, "Tables of Functions," Dover Publications, New York, N. Y., 1943, p. 147. 


$$
\begin{aligned}
J_{m}\left(\kappa_{n} r\right) & \left.=\left(j^{-m} / 2 \pi\right) \int_{0}^{2 \pi} e^{-j\left[\kappa_{n} r \cos \phi+m \phi\right.}\right] d \phi \\
& =\int_{ \pm \theta}^{2 \pi \pm \theta} e^{-j\left[\kappa_{n} r \cos (\beta \mp \theta)+m(\beta \mp \theta)-m \pi / 2\right]} d \beta .
\end{aligned}
$$

Hence

$$
\begin{aligned}
f(r, \theta) & =\int_{ \pm \theta}^{2 \pi \pm \theta} e^{-j k \sin \alpha \cos (\beta \mp \theta) r} d \beta \\
g(\beta) & =1 / 2 \pi e^{-j m(\beta+\pi / 2)}
\end{aligned}
$$

where $\alpha$ is given by (16). Expanding the cosine in the exponent of (27) and substituting in (23) we obtain

$$
\psi(r, \theta, z)=\int_{ \pm \theta}^{2 \pi \pm \theta} g(\beta) e^{-j \bar{K} \cdot \bar{R}} d \beta
$$

where $\bar{K}$ and $\bar{R}$ are given by (19) and (20).

The solution given by (29) represents an infinite manifold of plane waves, having amplitudes $d \beta / 2 \pi$ and phase $2 \pi g(\beta)$ propagating along coaxial cones of aperture $2 \alpha$ and at azimuthal angles $\beta$ with the velocity of light in the medium. The situation is more complex than in the case of the rectangular guide since the number of waves required for the resolution of a guided wave is infinite, but since the angle $\alpha$ is given by (16) in both cases, the phase velocity, the group velocity, and the phenomenon of cutoff can all be interpreted as in the case of the rectangular guide.

As an example of plane-wave resolution in a circular guide, we choose a vertically polarized $H_{11}$ wave, since this mode has the lowest cutoff frequency of all possible circular modes, traveling in the positive $z$ direction. From (3) and (6) we conclude that vertical polarization; i.e., no $\theta$ component of electric field at $\theta=\pi / 2$, requires the solutions of (23) to be combined in such a way to give $\psi$ varying as $\cos \theta$; hence we write

$$
\begin{gathered}
\psi_{H_{11}}(r, \theta, z)=1 / 2 J_{1}\left(\kappa_{1} r\right)\left[e^{j \theta}+e^{-j \theta}\right] e^{-j h z} \\
=1 / 2\left(\psi_{1}+\psi_{2}\right) \\
J_{1}^{\prime}\left(\kappa_{1} a\right)=0
\end{gathered}
$$

where $\quad \psi_{2}^{1}=\int_{ \pm 0}^{2 \pi \pm \theta}\left[\frac{j e^{-j \beta}}{2 \pi}\right] e^{-j k \sin \alpha \cos (\beta \mp \theta) r} d \beta$.

$\psi_{2}^{1}$ represent two infinite manifolds of plane waves of phase distribution $j e^{-j \beta}$ and amplitudes $1 / 2 \pi d \beta$, each manifold being constituted to effect the vanishing of the tangential electric field at $r=a$, and the two manifolds being combined to give a vertically polarized wave.

A similar treatment may be made for the solutions in a coaxial guide if the contour integral representation of the Neumann function is introduced along with the representation of the Bessel function given by (26). When the Bessel and Neumann functions are combined in such a way that, for the proper choice of the eigenvalue, the tangential electric field vanishes at both the inner and outer conductors, the resultant manifold of plane waves will be found to be reflected back and forth, while also progressing along the $z$ axis, between the outer and inner conductors.

\section{Appendix A}

\section{VECTOR Notation}

The co-ordinate systems used in the foregoing were rectangular (right-handed) and cylindrical polar $(\theta$ measured counterclockwise and $\theta=0$ when $\left.\bar{r}_{1} \equiv \bar{\imath}\right)$, and the corresponding vector co-ordinates are $(\bar{x}=i x, \bar{y}=j y$, $z=\bar{k} z)$ and $\left(\bar{r}=\bar{r}_{1} r, \bar{\theta}=\bar{\theta}_{1} \theta, \bar{z}=\bar{k} z\right)$ where $\bar{i}, \bar{j}, \bar{k}, \bar{r}_{1}$, and $\bar{\theta}_{1}$ are unit vectors in the positive $x, y, z, r$, and $\theta$ directions, respectively.

The gradient, or directional derivative of a scalar $V$ is given by

$$
\nabla V=\bar{\imath} \partial V / \partial x+\bar{\jmath} \partial V / \partial y+\bar{k} \partial V / \partial z
$$

which, in cylindrical polar co-ordinates, may be written

$$
\nabla V=\bar{r}_{1} \partial V / \partial r+\bar{\theta}_{1} / r \partial V / \partial \theta+\bar{k} \partial V / \partial z .
$$

The curl of a vector $\bar{V}\left(=\bar{\imath} V_{x}+\bar{j} V_{y}+\bar{k} V_{z}\right)$ is given by

$\nabla \times \bar{V}=i\left(\partial V_{z} / \partial y-\partial V_{y} / \partial z\right)+j\left(\partial V_{x} / \partial z-\partial V_{z} / \partial x\right)$

$$
+\bar{k}\left(\partial V_{y} / \partial x-\partial V_{x} / \partial y\right)
$$

which, in cylindrical polar co-ordinates, may be written

$$
\begin{aligned}
\nabla \times \bar{V}= & \left.\left.\bar{r}_{1}\left[\frac{1}{r} \frac{\partial V_{z}}{\partial \theta}-\frac{\partial V_{\theta}}{\partial z}\right]+\bar{\theta}_{1}\right] \frac{\partial V_{r}}{\partial z}-\frac{\partial V_{z}}{\partial r}\right] \\
& +\bar{k}\left[\frac{1}{r} \frac{\partial\left(r V_{s}\right)}{\partial r}-\frac{\partial V_{r}}{\partial \theta}\right] .
\end{aligned}
$$

\section{Appendix B}

\section{Solu'tions of the Scalar-Wave Equation}

The scalar-wave equation ${ }^{8}$ may be written

$$
\partial^{2} \psi / \partial x^{2}+\partial^{2} \psi / \partial y^{2}+\partial^{2} \psi / \partial z^{2}+k^{2} \psi=0 .
$$

The assumption of a cylindrical-co-ordinate system $(u, v, z)$ can be introduced by assuming a solution of the form (10), so that the solution of (37) is reduced to the solution of (11), which may be written

$$
\partial^{2} f / \partial x^{2}+\partial^{2} f / \partial y^{2}+\kappa^{2} f=0 .
$$

On rectangular co-ordinates the substitution of the trial solution $f(x, y)=X(x) Y(y)$ leads to the result

$f(x, y)=(A \cos \mu x+B \sin \mu x)(C \cos \nu y+D \sin \nu y)$

$$
\kappa^{2}=\mu^{2}+\nu^{2} \text {. }
$$

If the planes $x=0, x=a, y=0$, and $y=b$ are assumed perfectly conducting the boundary condition (8) requires

$$
\left.\frac{\partial \psi_{E}}{\partial x}\right|_{y=0}=\left.\frac{\partial \psi_{E}}{\partial x}\right|_{y=b}=\left.\frac{\partial \psi_{E}}{\partial y}\right|_{x=0}=\left.\frac{\partial \psi_{E}}{\partial y}\right|_{x=a}=0
$$

while (9) requires

$$
\left.\frac{\partial \psi_{H}}{\partial x}\right|_{x=0}=\left.\frac{\partial \psi_{H}}{\partial x}\right|_{x=a}=\left.\frac{\partial \psi_{H}}{\partial y}\right|_{y=0}=\left.\frac{\partial \psi_{H}}{\partial y}\right|_{y=b}=0 .
$$

From (10) it is seen that (41) and (42) may be applied directly to $f(u, v)$, in this case given by (39). Applying (41) to (39) we obtain

$$
f_{E}(x, y)=\sin \mu x \sin \nu y, \mu=m \pi / a, \nu=n \pi / b
$$

while applying (42) to (39) yields

$$
f_{H}(x, y)=\cos \mu x \cos \nu y, \mu=m \pi / a, \nu=n \pi / b \text {. }
$$

Substituting (43) and (44) in (10) yields (12), (13), and (14). 
In polar co-ordinates $(r, \theta)$, (38) becomes

$$
\partial^{2} f / \partial r^{2}+1 / r \partial f / \partial r+1 / r^{2} \partial^{2} f / \partial \theta^{2}+\kappa^{2} f=0 .
$$

The assumption of the trial solution $f(r, \theta)=R(r) \Theta(\theta)$ yields Bessel's equation for $R(r)$ and the harmonic equation for $\Theta(\theta)$ with the result

$$
f(r, \theta)=\left[A J_{m}(\kappa r)+B N_{m}(\kappa r)\right] e^{i( \pm m \theta+\phi)}
$$

where $J_{m}$ and $N_{m}$ are Bessel functions of the first and second kind, respectively, and $A, B$, and $\phi$ are arbitrary. Inasmuch as $N_{m}(0)$ is infinite, $B$ must be equated to zero for a solution in a hollow circular guide (this would not be true in a coaxial guide), while continuity of the solution with respect to $\theta$ demands that $m$ be an integer. Assuming the perfectly conducting boundary $r=a,(8)$ and (9) become

$$
\begin{aligned}
& \partial \psi_{E} /\left.\partial \theta\right|_{r=a}=0 . \\
& \partial \psi_{H} /\left.\partial r\right|_{r=a}=0 .
\end{aligned}
$$

Applying these conditions to (46), the transcendental equations (24) and (25) are obtained, where the integer $n$ denotes the sequence of the roots to the equations starting with the smallest in each case.

By convention, $n$ runs from one to infinity, and there is no $n=0$ root.

\title{
Note on the Measurement of Transformer Turns-Ratio*
}

\author{
P. M. HONNELL $\dagger$, SENIOR MEMBER, I.R.E.
}

Summary-This note shows that in many important cases the turns-ratio of iron-core transformers is given by the simple relation

$$
N_{1} / N_{2}=\sqrt{X_{s c 1} / X_{s c 2}},
$$

in which $X_{s c 1}$ and $X_{s c 2}$ are the reactive components of the shortcircuit primary and secondary impedance of the windings concerned. The measurements are conveniently made with an impedance or inductance bridge. This equation also gives the turns-ratio of certain air-core transformers.

\section{INTRODUCTION}

$\prod$

HE RATIO of turns of its windings is probably the most important parameter of a transformer; and the occasion for occurrence.

The procedure ${ }^{1-4}$ most commonly given for determining this parameter is that of exciting the primary winding by a known alter-

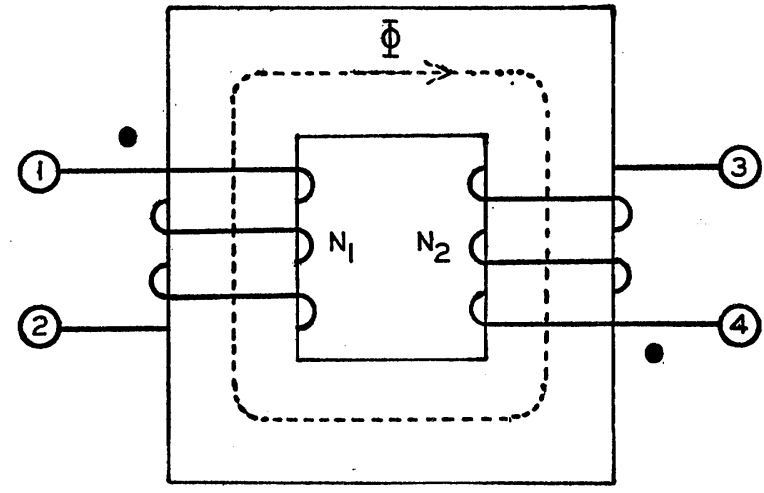

Fig. 1-The iron-core transformer.

nating voltage, and measuring the secondary winding open-circuit voltage by means of a high-impedance voltmeter. The turns-ratio is then taken as equal to the ratio of the terminal voltages of the windings. Although this method is very useful, particularly with power transformers, its accuracy is seriously limited by leakage inductance

* Decimal classification: R264.3. Original manuscript received by the Institute, April 17, 1945; revised manuscript received, June 21, 1945.

Signal Corps, U. S. Miltary Academy, West Point. New York.

1 F. E. Terman, "Radio Engineers' Handbook," first edition, McGraw-Hill Book Company, New York 18 , N. Y., 1943, p. 973.

2 A. E. Knowlton, "Standard Handbook for Electrical Engineers," McGraw-Hill Book Company, New York 18, N. Y., 1941, p. 611.

3 H. Pender and K. Mcllwain, "Electrical Engineers' Handbook," John Wiley and Sons, Inc., New York, N. Y., 1941, pp. 7-23, section 7.

4 Electrical Engineering Staff, Massachusetts Institute of Technology, "Mag tic Circuits and Transformers," John Wiley and Sons, Inc., New York, N. Y., 1943, pp. $442-448,352-353$ and distributed capacitance in the windings, and by instrumentation This is particularly true when use is made of vacuum-tube voltmeters (to minimize the secondary burden) in the measurement of audiofrequency transformers. Even if not subject to these errors, this method is only an approximation, since it is strictly a measure of the ratio of the transformer mutual impedance to primary impedance, and not a measurement of the turns-ratio.

Precision methods of calibrating instrument-transformer ratios ${ }^{5}$ are likewise concerned only with determinations of terminal voltage or current ratios, and are also not measurements of the actual turnsratio.

The purpose of this paper is to point out the simplicity of determining the turns-ratio by measuring the transformer short-circuit impedances from both the primary winding (giving $Z_{s c 1}$ ) and the secondary winding (giving $Z_{s c 2}$ ). These measurements are conveniently made on an impedance or inductance bridge. The desired transformer turns-ratio $N_{1} / N_{2}$ is given to a high order of accuracy by the square

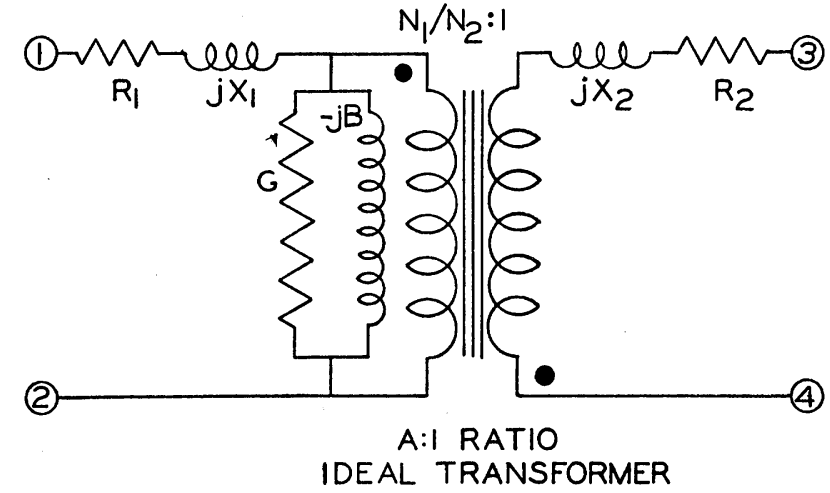

Fig. 2-An equivalent circuit representing the iron-core transformer of Fig. 1.

root of the quotient of the reactive (or inductive) components of the short-circuit impedances; that is,

$$
N_{1} / N_{2}=\sqrt{X_{s c 1} / X_{s c 2}}=\sqrt{L_{s c 1} / L_{s c 2}}
$$

This method - for all its ease of application and high accuracy -is apparently not well known.

\section{The Iron-Core Transformer}

The basis for the application of (1) to an iron-core transformersuch as shown in Fig. 1-may be established from a consideration of one of its equivalent circuits, shown in Fig. 2. In the latter illustration,

${ }^{5}$ F. A. Laws, "Electrical Measurements," Second Edition, McGraw-Hill Book Company, Inc., New York, 1938, pp. 609-622. 